\title{
Complete thoracoscopic sleeve lobectomy for mucoepidermoid carcinoma in a 5-year-old boy
}

\author{
Xukai Li $^{1}$, Tuo Xing ${ }^{1}$, Fei Cui ${ }^{2}$, Jianxing $\mathrm{He}^{1}$ \\ ${ }^{1}$ Department of Thoracic Surgery and Oncology, ${ }^{2}$ Department of Cardiothoracic Surgery, The First Affiliated Hospital of Guangzhou Medical \\ University, Guangzhou Institute of Respiratory Disease, Guangzhou 510120, China \\ Correspondence to: Jianxing He, PhD, MD. Department of Thoracic Surgery/Oncology, the First Affiliated Hospital of Guangzhou Medical \\ University, Guangzhou Institute of Respiratory Disease, Guangzhou 510120, China. Email: drjianxing.he@gmail.com.
}

Submitted Jul 17, 2019. Accepted for publication Aug 12, 2019.

doi: $10.21037 /$ jtd.2019.08.91

View this article at: http://dx.doi.org/10.21037/jtd.2019.08.91

\section{Introduction}

Mucoepidermoid carcinoma (MEC) is a salivary gland tumor characterized by parotid differentiation (1). Pulmonary mucoepidermoid carcinoma (PMEC) originates from the small salivary glands of the bronchial trachea. PMEC is a kind of uncommon primary lung cancer with low incidence, accounts for about $0.1-1.0 \%$ of lung carcinoma $(2,3)$, especially rare in children. Radical resection is considered as the best treatment modality due to its good prognosis results.

\section{Case presentation}

A 5-year-old boy was admitted to the pediatric department of our hospital due to recurrent fever, cough and hemolytic sputum for more than 1 year. A high-resolution computed tomography (HRCT) showed a mass in the left hilum of the lung and lead to atelectasis on the left lower lobe, a nodule in the left lower lobe orifice (Figure 1). After admission, bronchoscopic biopsy was applied and pathology examination was MEC. Pulmonary ventilation perfusion showed that left upper lobe accounted for $28.1 \%$, the lower left lung accounted for only $6.3 \%$.

To minimize trauma and preserve the lung function as much as possible, we performed minimally invasive left lower lung sleeve resection under general anesthesia. The right main bronchus was intubated with a $4.5 \mathrm{~mm}$ single lumen endobronchial tube to maintain right singlelung ventilation. Ventilator parameters were set as $\mathrm{FiO}_{2}$ 0.5 , TV $140 \mathrm{~mL}$, RR 15 times/min. We used the standard 3 -port approach, the first $1 \mathrm{~cm}$ incision was chosen in the sixth intercostal space of the preaxillary line for the camera (30 degree $4 \mathrm{~mm}$ HD video thoracoscope). The second $3 \mathrm{~cm}$ incision was selected in the fourth intercostal space in the preaxillary line as the main operating hole. The third $0.5 \mathrm{~cm}$ incision was made in the sixth intercostal space in the post axillary line. During the operation, a $3.5 \mathrm{~cm}$ round tumor was found in the left lower lobe opening and protruded into the left main stem bronchus. The lumen of the left upper bronchus was smooth, leading us to perform a left lower sleeve lobectomy. The surgical margins of the left main bronchus and upper left bronchus were reported to be tumor-free according to the quick frozen sections evaluation. The residual left main bronchus was anastomosed with the left upper bronchus continuously (Figure 2), end to end reinforced anastomosis subsequently and ensure no air leaks. Finally, hilar and mediastinal lymph nodes were dissected (Figure 3).

The pathological report confirmed that the tumor was T2aN0M0 (stage IB) MEC (Figure 4). No short-term complications were occurred. Reexamination with the bronchoscope at 40 days later and chest CT at 4 months later showed that the anastomotic site was normal without any stenosis (Figure 5).

\section{Discussion}

MECs are classified into low grade malignant MECs and high grade malignant MECs based on the differentiation degree and biological behavior of cancer cells. Low-grade tumor cystic changes account for the main components, and there are mucinous secretory cells, squamous or squamous cells and 

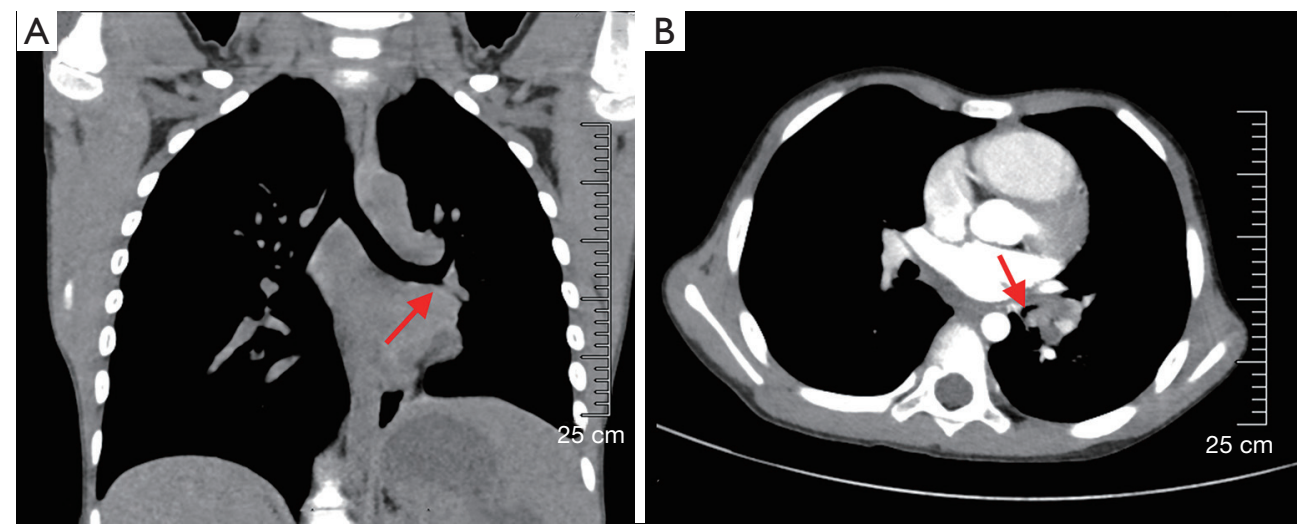

Figure 1 Preoperative chest computed tomography (CT) images showing a tumor in left lower lobe. (A) $30 \mathrm{~mm} \times 20 \mathrm{~mm}$ mass was presented in the left hilum (arrow) and left lung volume decreased; (B) endobronchial nodules were found in the left lower lobe orifice (arrow).

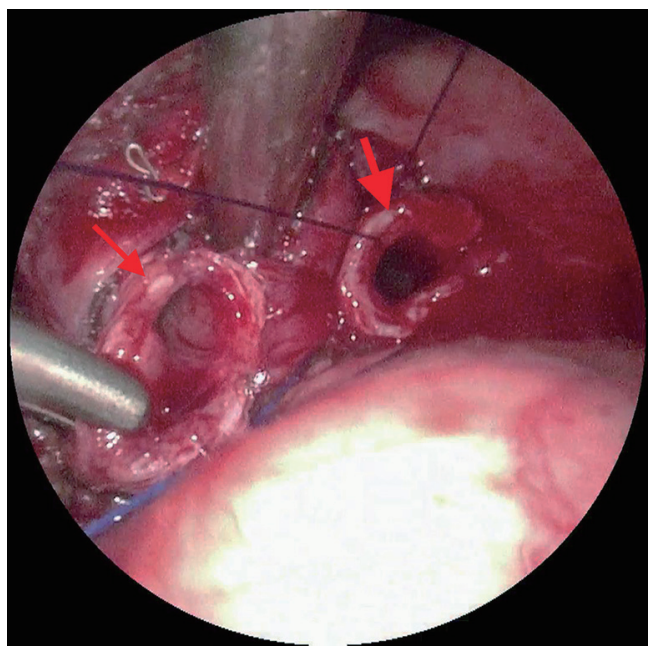

Figure 2 Anastomosis was performed between the left superior bronchus (small arrow) and the left main bronchus (large arrow).

intermediate cells composed of different morphological structures. High-grade MECs are rare, most of which are intermediate cells and squamous cells, with few secreting cells, heterogeneous nuclei, high proportion of cytoplasm and easy mitotic images (5). PMEC is characterized by slow growth, atypical symptoms (cough, hemoptysis, wheezing, and obstructive pneumonia) (6), and imaging findings, thereby resulting in about 1 year delay for the diagnosis.

Most salivary gland-type lung tumors are indolent, Regional radical resection is considered to be the most effective treatment, no matter lymph node metastasis (7). The conventional surgical approach is the posterolateral thoracotomy. Advances thoracoscopy lead video-assisted

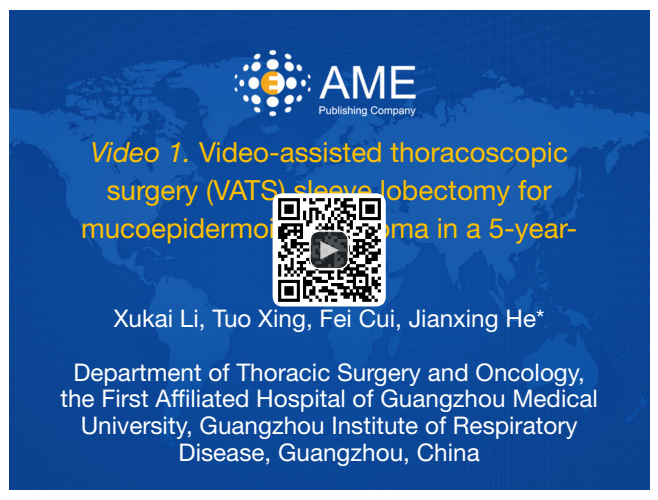

Figure 3 Video-assisted thoracoscopic surgery (VATS) sleeve lobectomy for mucoepidermoid carcinoma in a 5 -year-old boy (4). Available online: http://www.asvide.com/watch/32948

thoracoscopic surgery (VATS) to the mainstream. Surgery methods for PMECs treatment include bronchotomy with excision of tumor, sleeve resection, lobectomy or pneumonectomy, which could be performed under videoassisted thoracoscopy in adults. However, small chest space, narrow intercostal space, and difficulties in one-lung ventilation were presented in pediatric patients, which could result in techniques barrier during thoracoscopic surgery, especially lobectomy and sleeve resection. The operative anastomosis is foremost for this operation, the choice of incision is crucial, instruments entered through two incisions were perpendicular to the hilum, make it more beneficial to expose blood vessels and bronchus. Standard 4-mm instrument in this child could provide enough assistance to complete this procedure via a VATS approach.

Santambrogio et al. reported the first case of video- 


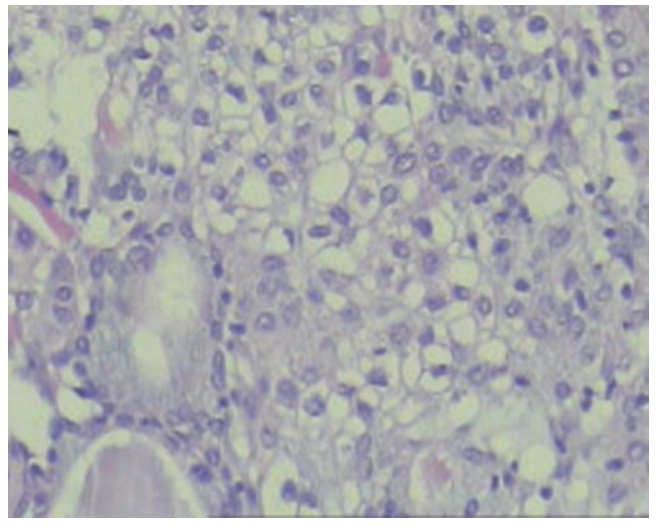

Figure 4 Low-grade mucoepidermoid carcinoma was confirmed by the pathological examination under a light microscope $(H \& E \times 400)$.

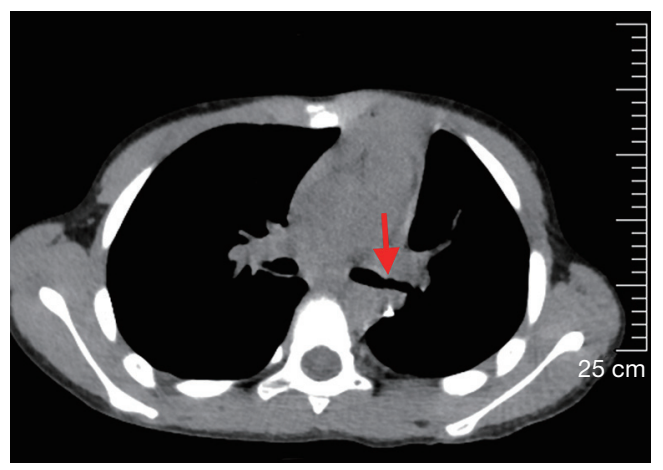

Figure 5 Smooth presentation without any stenosis was found on the bronchial anastomosis by CT (arrow).

assisted sleeve resection of the left lower lobe bronchus in a 15 -year-old girl (8). Hence, to the best of our knowledge, this is the PMEC tumor with youngest age treated with complete thoracoscopic sleeve lobectomy.

\section{Conclusions}

In conclusion, thoracoscopic sleeve lobectomy is technically difficult, but feasible, even in the first 5 years of life. The operation can be favourably performed by experienced senior thoracoscopic surgeons.

\section{Acknowledgments}

None.

\section{Footnote}

Conflicts of Interest: The authors have no conflicts of interest to declare.

Ethical Statement: The authors are accountable for all aspects of the work in ensuring that questions related to the accuracy or integrity of any part of the work are appropriately investigated and resolved. Written informed consent was obtained from the patient for publication of this manuscript and any accompanying images.

\section{References}

1. Molina JR, Aubry MC, Lewis JE, et al. Primary salivary gland-type lung cancer: spectrum of clinical presentation, histopathologic and prognostic factors. Cancer 2007;110:2253-9.

2. Moran CA. Primary salivary gland-type tumors of the lung. Semin Diagn Pathol 1995;12:106-22.

3. Heitmiller RF, Mathisen DJ, Ferry JA, et al. Mucoepidermoid lung tumors. Ann Thorac Surg 1989;47:394-9.

4. Li X, Xing T, Cui F, et al. Video-assisted thoracoscopic surgery (VATS) sleeve lobectomy for mucoepidermoid carcinoma in a 5-year-old boy. Asvide 2019;6:263. Available online: http://www.asvide.com/watch/32948

5. Lichtenberger JP 3rd, Biko DM, Carter BW, et al. Primary Lung Tumors in Children: Radiologic-Pathologic Correlation From the Radiologic Pathology Archives. Radiographics 2018;38:2151-72.

6. Madan K, Agarwal R, Bal A, et al. Bronchoscopic management of a rare benign endobronchial tumor. Rev Port Pneumol 2012;18:251-4.

7. Kang DY, Yoon YS, Kim HK, et al. Primary salivary gland-type lung cancer: surgical outcomes. Lung Cancer 2011;72:250-4.

8. Santambrogio L, Cioffi U, De Simone M, et al. Videoassisted sleeve lobectomy for mucoepidermoid carcinoma of the left lower lobar bronchus: a case report. Chest 2002;121:635-6.

Cite this article as: Li X, Xing T, Cui F, He J. Complete thoracoscopic sleeve lobectomy for mucoepidermoid carcinoma in a 5-year-old boy. J Thorac Dis 2019;11(9):4028-4030. doi: 10.21037/jtd.2019.08.91 